\title{
Diagnostic accuracy of a serotype-specific antigen test in community-acquired pneumonia
}

\author{
Susanne M. Huijts' ${ }^{1}$ Michael W. Pride ${ }^{2}$, Josephine M.I. Vos ${ }^{3}$, Kathrin U. Jansen², \\ Chris Webber ${ }^{4}$, William Gruber ${ }^{5}$, Wim G. Boersma 6 , Dominic Snijders 6 , \\ Jan A.J.W. Kluytmans ${ }^{7}$, Ivo van der Lee ${ }^{8}$, Bart A.F. Kuipers ${ }^{9}$, \\ Arie van der Ende ${ }^{10,11}$ and Marc J.M. Bonten ${ }^{1,12}$
}

\begin{abstract}
Affiliations: 'Julius Center for Health Sciences and Primary Care, University Medical Center Utrecht, Utrecht, ${ }^{3}$ Dept of Internal Medicine/Haematology, St Antonius Hospital, Nieuwegein, ${ }^{6}$ Dept of Pulmonary Diseases, Medical Centre Alkmaar, Alkmaar, ${ }^{7}$ Laboratory for Microbiology and Infection Control, Amphia Hospital, Breda, ${ }^{8}$ Dept of Pulmonology, Spaarne Hospital, Hoofddorp, ${ }^{9}$ Pulmonary Diseases, Isala Klinieken, Zwolle, ${ }^{10}$ Dept of Medical Microbiology, Academic Medical Centre, Amsterdam, ${ }^{11}$ Netherlands Reference Laboratory for Bacterial Meningitis, Academic Medical Centre, Amsterdam, and ${ }^{12}$ Dept of Medical Microbiology, University Medical Center Utrecht, Utrecht, The Netherlands. ${ }^{2}$ Pfizer Vaccines Research, Pearl River, NY, and ${ }^{5}$ Pfizer Vaccine Clinical Research, New York, NY, USA. ${ }^{2}$ ffizer Vaccine Research, Maidenhead, UK.
\end{abstract}

Correspondence: S.M. Huijts, UMC Utrecht/Julius Center for Health Sciences and Primary Care, Stratenum 6.131, Room 6.125, P.0. Box 85500, 3508 GA Utrecht, The Netherlands. E-mail: s.m.huijtsqumcutrecht.nl

ABSTRACT Our aim was to evaluate the diagnostic accuracy and clinical utility of a serotype-specific urinary antigen detection multiplex assay for identification of 13 pneumococcal serotypes $(1,3,4,5,6 \mathrm{~A}, 6 \mathrm{~B}$, $7 \mathrm{~F}, 9 \mathrm{~V}, 14,18 \mathrm{C}, 19 \mathrm{~A}, 19 \mathrm{~F}$ and $23 \mathrm{~F}$ ) in urine of patients with community-acquired pneumonia.

Adult patients with clinical suspicion of community-acquired pneumonia were included. In addition to standard diagnostic procedures, a urine sample was collected to perform the urinary antigen detection test. Demographic, clinical, radiological and microbiological data were collected.

Among 1095 community-acquired pneumonia patients Streptococcus pneumoniae was identified as causative pathogen in 257 (23\%), when using conventional diagnostic methods and in 357 (33\%) when urinary antigen detection was added. Of the 49 bacteraemic episodes caused by one of the 13 serotypes covered by the urinary antigen detection, 48 were detected by the urinary antigen detection, indicating a sensitivity of $98 \%$. Of the 77 community-acquired pneumonia episodes with a "non-urinary antigen detection" causative pathogen, none had a positive urinary antigen detection result, indicating a specificity of $100 \%$.

Addition of the urinary antigen detection test to conventional diagnostic methods increased the prevalence of S. pneumoniae community-acquired pneumonia by $39 \%$. Using bacteraemic episodes as reference sensitivity and specificity of the urinary antigen detection was $98 \%$ and $100 \%$, respectively.

@ERSpublications

Addition of urinary antigen detection test to conventional diagnostic methods increases prevalence of S. pneumoniae CAP http://ow.ly/nSA1l

Received: Aug 302012 | Accepted after revision: Jan 152013 | First published online: Feb 082013

Support statement: This study was sponsored by Wyeth Pharmaceuticals, Inc., which was acquired by Pfizer in October 2009.

Conflict of interest: Disclosures can be found alongside the online version of this article at www.erj.ersjournals.com

Copyright @ERS 2013 


\section{Introduction}

Community-acquired pneumonia (CAP) is a major cause of morbidity and mortality worldwide. Streptococcus pneumoniae is the most common causative organism of CAP in all age groups [1,2]. There are $>90$ different serotypes of $S$. pneumoniae, based on capsular polysaccharides, with marked serotype-specific differences in disease prevalence [3] and clinical outcome of invasive pneumococcal disease [4].

The diagnosis of pneumococcal CAP is usually based on clinical and radiographic evidence of pneumonia combined with microbiological results of blood and sputum samples, and detection of pneumococcal antigens in urine. However, many episodes of pneumococcal CAP will remain undiagnosed. Bacteraemia with S. pneumoniae is demonstrated in $3.6-9.6 \%$ of the adult cases with CAP only [5], and sensitivity decreases due to prior use of antibiotics $[6,7]$. Furthermore, only $36-64 \%$ of CAP patients produce sputum $[8,9]$, and samples are frequently contaminated with flora of the upper respiratory tract. Finally, detection of pneumococcal antigens in urine, usually with the immunochromatographic assay (ICA) of BinaxNOW (Alere International, Galway, Ireland), may have a high specificity, yet reported sensitivities have varied from $76.9-100 \%$ in the subgroup of patients with pneumococci isolated from sterile sites (such as blood and pleural fluid) and from 43.7-69.2\% in patients with pneumococci isolated in sputum only [10-12]. Serotyping is only possible for pneumococci isolated from sterile sites and sputum. As a result, serotype distributions and changes herein (for instance, because of vaccination strategies) can only be measured in a small subset of CAP cases.

In preparation for a large randomised placebo-controlled double-blind trial [13], we evaluated the diagnostic properties and clinical utility of a novel serotype-specific urinary antigen detection (UAD) multiplex assay [14] for the identification of 13 serotype-specific polysaccharides of S. pneumoniae (serotypes 1, 3, 4, 5, 6A, 6B, 7F, 9V, 14, 18C, 19A, 19F and 23F) in human urine samples in patients hospitalised with CAP. The 13 polysaccharide antigens correspond to the serotypes used in the 13-valent pneumococcal conjugate vaccine.

\section{Material and methods \\ Patients}

We conducted a prospective, observational, cohort study in 23 Dutch hospitals (four academic, 15 teaching and four nonteaching hospitals), between January 2008 and April 2009. Not all hospitals started at the same time (the last one started in July 2008) and 17 hospitals discontinued patient enrolment in October 2008 because of a competing pneumonia trial.

Adult patients aged $\geqslant 18$ years with a clinical suspicion of CAP or lower respiratory tract infection (LRTI) presenting at the emergency room of the participating hospitals were eligible. A clinical suspicion of CAP or LRTI was defined as the presence of at least two of the following criteria: fever or hypothermia; cough or change in chronic coughing pattern; dyspnoea, tachypnoea or hypoxia; findings with percussion or auscultation consistent with pneumonia; leukocytosis, leukopenia, left shift or an infiltrate on the chest radiograph. In each hospital, dedicated research nurses checked admission diagnoses of patients admitted through the emergency room two to three times weekly. Eligible patients not included at the emergency room could be included for up to $48 \mathrm{~h}$ after admission. The study was approved by all local Research Ethics Committees and written informed consent was obtained from all participants.

\section{Diagnostic approach}

In addition to history taking, physical examination, laboratory blood analysis and chest radiograph, diagnostic procedures included microbiological cultures of blood, sputum and pleural fluid (if present), and collection of a urine sample immediately after presentation, or at least within $48 \mathrm{~h}$ of admission.

Urine samples were processed locally in a standardised manner (treated with 0.5 M PIPES buffer to a final concentration of $25 \mathrm{mM}$ ) and subsequently frozen at $-70^{\circ} \mathrm{C}$ until processing in the reference laboratory of Pfizer Vaccine Research (Pearl River, NY, USA). As ICA was not part of standard care in most hospitals, local processing of ICA was not part of study protocol. In the reference laboratory, both the UAD test and the commercially available urinary pneumococcal antigen ICA (BinaxNOW S. pneumoniae urine antigen test, Alere International) were performed batch-wise according to manufacturers' instructions. The ICA results were interpreted by two analysts, blinded for any clinical information. A third analyst interpreted the results when the first two analysts did not agree. The UAD test is a Luminex technology-based multiplex UAD assay, which can detect simultaneously 13 different serotypes of $S$. pneumoniae (serotypes 1, 3, 4, 5, $6 \mathrm{~A}, 6 \mathrm{~B}, 7 \mathrm{~F}, 9 \mathrm{~V}, 14,18 \mathrm{C}, 19 \mathrm{~A}, 19 \mathrm{~F}$ and $23 \mathrm{~F}$ ) by capturing serotype-specific polysaccharides secreted in human urine [14]. The in vitro validation of the test (based on a subset of the current study population) and specificity determination in noninfected individuals has been reported elsewhere [14]. 
Microbiological testing (blood and sputum cultures and BinaxNOW urinary Legionella antigen test, if clinically applicable) was performed in local laboratories according to local and manufacturers' protocols. S. pneumoniae blood isolates were serotyped at the Netherlands Reference Laboratory for Bacterial Meningitis, (Amsterdam, the Netherlands) by co-agglutination and subtyping with the capsular swelling method (the Quellung reaction) using antisera (Statens Serum Institute, Copenhagen, Denmark) according to the manufacturer's protocol $[15,16]$. Coagulase-negative staphylococci in blood cultures were considered as "contaminants" and not as a causative pathogen.

\section{Data collection}

Demographics, prior antibiotic use, signs and symptoms of infection, radiological and microbiological test results and findings of physical examination were documented in a standardised case record form by trained research nurses and/or physicians in every hospital. Data were collected during or shortly after admission. Clinical severity of CAP was categorised upon the Pneumonia Severity Index (PSI) score [17].

\section{Definitions}

CAP was defined as the presence of an infiltrate on the chest radiograph within $48 \mathrm{~h}$ of admission based on the diagnosis of the local radiologist together with at least two of the following signs or symptoms: cough, sputum production, fever, auscultatory findings consistent with pneumonia, leukocytosis or leukpenia, C-reactive protein $>3$ times the upper limit of normal, hypoxaemia with oxygen tension $<60 \mathrm{mmHg}$ while patient was breathing room air or signs of dyspnoea/tachypnoea.

The causative microorganism of CAP was considered "definite" if it was cultured from blood or any other sterile body fluid or if the urinary antigen test was positive (either for pneumococcal or Legionella antigen with ICA or with the UAD test). Bacteria considered as contaminants (e.g. coagulase-negative staphylococci) were not considered as causative pathogens. A microorganism was considered a "probable" cause of CAP in the absence of a definite pathogen and when it was present as dominant flora in the sputum culture. Pneumococcal CAP was defined as CAP with S. pneumoniae as the "definite" or "probable" causative microorganism.

\section{Data analysis}

Descriptive analyses were carried out by calculating frequencies, mean or median with SPSS statistical package (version 17.0, SPSS Inc., Chicago, IL, USA). To compare groups, the Pearson's Chi-squared test was used for dichotomous determinants and the t-test or Mann-Whitney U-test was used for continuous data. A p-value $<0.05$ was considered significant.

To calculate the sensitivity of the UAD test, "true positives" were defined as CAP with bacteraemia (blood culture positive) caused by one of the 13 serotypes of the UAD test and the number of positive UAD tests was compared with the number of "true positives". Episodes of probable pneumococcal CAP that were based on sputum cultures only were excluded from this analysis. "True negatives" were defined as CAP cases with bacteraemia caused by another pathogen or by pneumococcal serotypes not covered by the UAD test or CAP cases with only a positive Legionella urinary antigen test. Specificity was determined by the number of negative UAD tests compared with the number of "true negatives".

\section{Results \\ Study population}

In total, 1758 patients with a clinical suspicion of CAP or LRTI were included, of whom 552 (31\%) had no infiltrate on chest radiograph within $48 \mathrm{~h}$ of admission. Urine samples had not been obtained from 101 patients and informed consent was not obtained from 10 patients, yielding a total study population of 1095 patients with CAP (fig. 1). The median age of these 1095 patients was 69 years (interquartile range $57-79$ years), the majority were male $(62.7 \%)$ and $50(4.6 \%)$ patients were treated as outpatients. Median duration of complaints before presentation was 4 days (interquartile range 2-7 days). PSI categorisation yielded a distribution of $9.9 \%, 22.1 \%, 23.0 \%, 34.6 \%$ and $10.4 \%$ for categories I-V, respectively. Four $(0.4 \%)$ patients reported receipt of pneumococcal vaccination. Information on pre-admission antibiotic use was available for 1077 patients and 325 had received antibiotics before admission.

\section{Aetiology}

Blood cultures were available in $922(84.2 \%)$ patients, sputum cultures in $570(52.1 \%)$ patients and Legionella urinary antigen tests in $762(69.6 \%)$ patients. All 1095 urine samples were tested with ICA and $\mathrm{UAD}$ in the reference laboratory. There were three CAP episodes with urine antigen tests positive for both pneumococci and Legionella. In all three episodes, both ICA and UAD test were positive and, in one episode, pneumococcal bacteraemia was also present. S. pneumoniae was considered the causative pathogen 
1758 included

subjects with

suspected CAP

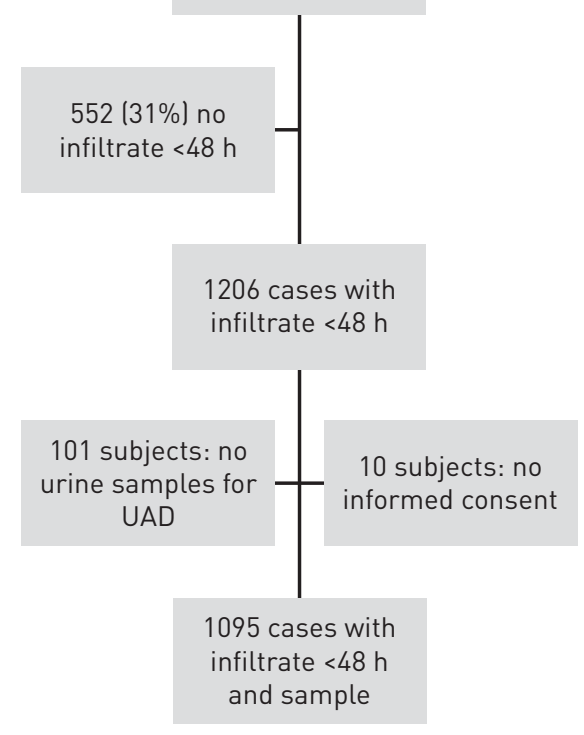

FIGURE 1 Flow chart showing the study population with suspected community-acquired pneumonia (CAP). UAD: urinary antigen detection.

of CAP in all three episodes. One CAP episode was associated with Escherichia coli bacteraemia, a positive ICA pneumococcal urinary antigen test and a negative UAD test. In this episode, S. pneumoniae was considered as the causative pathogen of CAP.

Based on microbiological culture results and ICA, an aetiological cause of CAP could be determined in 403 (36.8\%) episodes (table 1 ). When the UAD test results were added to these methods, aetiology could be determined in $493(45.0 \%)$ episodes, and proportions of episodes of pneumococcal CAP were $23.5 \%$ $(\mathrm{n}=257)$ and $32.6 \%(\mathrm{n}=357)$ without and with, respectively, inclusion of UAD test results. This represents a relative increase of the diagnostic yield for S. pneumoniae of $39 \%$ and an absolute increase of $9.1 \%$.

\section{TABLE 1 Aetiology of 1095 patients with confirmed community-acquired pneumonia}

\section{Pathogen}

Conventional diagnostics ${ }^{\#}$

\begin{tabular}{lll}
\hline $\begin{array}{l}\text { Definite } \\
\text { pathogen }\end{array}$ & $\begin{array}{l}\text { Probable } \\
\text { pathogen }\end{array}$ & Total $^{f}$ \\
\end{tabular}

Conventional diagnostics + UAD

\begin{tabular}{ccc}
\hline $\begin{array}{c}\text { Definite } \\
\text { pathogen }\end{array}$ & $\begin{array}{c}\text { Probable } \\
\text { pathogen }\end{array}$ & Total $^{f}$ \\
347 & 10 & $357(32.6)$ \\
1 & 34 & $35(3.2)$ \\
25 & & $25(2.3)$ \\
4 & 7 & $11(1.0)$ \\
& 7 & $7(0.6)$ \\
3 & 8 & $11(1.0)$ \\
5 & 6 & $11(1.0)$ \\
3 & 4 & $7(0.6)$ \\
11 & 18 & $29(2.6)$ \\
& & $602(55.0)$
\end{tabular}

Streptococcus pneumoniae
Haemophilus influenzae
Legionella pneumophila
Pseudomonas aeruginosa
Moraxella catarrhalis
Klebsiella spp.
Escherichia coli
Staphylococcus aureus
Other
Unknown

$\begin{array}{cccc}240 & 17 & 257(23.5) & 347 \\ 1 & 37 & 38(3.5) & 1 \\ 25 & & 25(2.3) & 25 \\ 4 & 11 & 15(1.4) & 4 \\ & 8 & 8(0.7) & \\ 3 & 9 & 12(1.1) & 3 \\ 5 & 6 & 11(1.0) & 5 \\ 3 & 4 & 7(0.6) & 3 \\ 11 & 19 & 30(2.7) & 11 \\ & & 692(63.2) & \end{array}$

Data are presented as $\mathrm{n}$ or $\mathrm{n}(\%)$. UAD: urinary antigen detection. ${ }^{*}$ : causative pathogen based on the results of blood or other sterile fluid culture, immunochromatographic assay (ICA) urinary antigen test only and sputum culture; ": causative pathogen based on results of blood or other sterile fluid culture, ICA urinary antigen test and UAD test results and sputum culture; ${ }^{+}$: pathogen cultured from blood or any other sterile body fluid or if the urinary antigen test was positive; ${ }^{\S}$ : pathogen identified in the absence of a definite pathogen and if it was present as dominant flora in the sputum culture; ${ }^{f}: \mathrm{n}=1095$. 
Diagnostic accuracy

In all, 249 (22.7\%) UAD tests and 211(19.3\%) ICA tests were positive in the study population. Of the 249 UAD-positive samples, 122 samples were negative in the ICA.

There were 49 bacteraemic isolates that belonged to one of the 13 serotypes included in the UAD test, of which 48 were detected by UAD, yielding a sensitivity of $98 \%$ for bacteraemic pneumococcal CAP with serotypes included in the UAD assay (table 2). The only negative urine sample yielded a positive signal that did not reach the predefined positivity cut-off limit for serotype 3, which was the serotype of the blood culture isolate. The ICA yielded positive results in 34 of these 49 episodes, yielding a sensitivity of $69.4 \%$ for the bacteraemic CAP with one of the 13 pneumococcal serotypes included in the UAD assay. The ICA detected 49 of the total of 77 bacteraemic pneumococcal CAP cases (including the serotypes not included in the UAD), indicating an overall sensitivity of $63.6 \%$.

There were 76 CAP episodes with causative pathogens other than S. pneumoniae serotypes included in the UAD test: 23 bacteraemic episodes due to non-UAD test $S$. pneumoniae serotypes, 28 nonpneumococcal bacteraemic cases and 25 episodes with mono-infection caused by Legionella pneumophila. There was no positive UAD test in this cohort, indicating an UAD assay specificity of $100 \%$. Of the 28 nonpneumococcal bacteraemic cases and the 25 episodes caused by L. pneumophila, there was one positive ICA test, indicating a specificity of $98 \%$.

\section{Serotype distribution}

Among the 77 episodes of bacteraemic pneumococcal CAP, serotypes 1 and 14 were the most common serotypes $(n=9)$, followed by $19 A$ and $7 F(n=7)$ and $22 F(n=6)$. Five isolates could not be serotyped. Based on the UAD results, serotype distribution of the 13 serotypes included appeared to change (fig. 2). Serotype 1 remained the most frequent serotype $(n=38)$ (table 3 ), but the relative frequencies of serotypes 3 and $18 \mathrm{C}$ increased, whereas that of serotype 14 decreased (from $18.4 \%$ (95\% confidence interval 7.5-29.2\%) to $8.4 \%$ (4.7-11.3\%) among bacteraemia isolates).

Based on the UAD results, 11 eleven CAP episodes (4.4\% of pneumococcal CAP cases diagnosed by UAD, $1.0 \%$ of all samples tested) appeared to be caused by multiple serotypes, which was confirmed by retesting and inhibition experiments (data not shown [14]). 10 episodes were caused by two serotypes included in the

TABLE 2 Results of urinary antigen detection (UAD) assay and immunochromatographic assay (ICA) in patients with bacteraemic and nonbacteraemic pneumococcal community-acquired pneumonia

\begin{tabular}{|c|c|c|c|}
\hline & \multicolumn{2}{|c|}{ UAD } & \multirow[t]{2}{*}{ Total } \\
\hline & Negative & Positive & \\
\hline \multicolumn{4}{|c|}{ BC negative for Streptococcus pneumoniae } \\
\hline ICA negative & 749 & 107 & 856 \\
\hline ICA positive & 71 & 91 & 162 \\
\hline Total & 820 & 198 & 1018 \\
\hline \multicolumn{4}{|c|}{ BC positive for $S$. pneumoniae, UAD serotype } \\
\hline ICA negative & 1 & 14 & 15 \\
\hline ICA positive & 0 & 34 & 34 \\
\hline Total & 1 & 48 & 49 \\
\hline \multicolumn{4}{|c|}{ BC positive for S. pneumoniae, non-UAD serotype } \\
\hline ICA negative & 12 & 0 & 12 \\
\hline ICA positive & 11 & 0 & 11 \\
\hline Total & 23 & 0 & 23 \\
\hline \multicolumn{4}{|c|}{ BC positive for $S$. pneumoniae, non-typeable serotype } \\
\hline ICA negative & 0 & 1 & 1 \\
\hline ICA positive & 2 & 2 & 4 \\
\hline Total & 2 & 3 & 5 \\
\hline \multicolumn{4}{|l|}{ Total } \\
\hline ICA negative & 762 & 122 & 884 \\
\hline ICA positive & 84 & 127 & 211 \\
\hline Total & 846 & 249 & 1095 \\
\hline
\end{tabular}

Data are presented as n. BC: blood culture. 


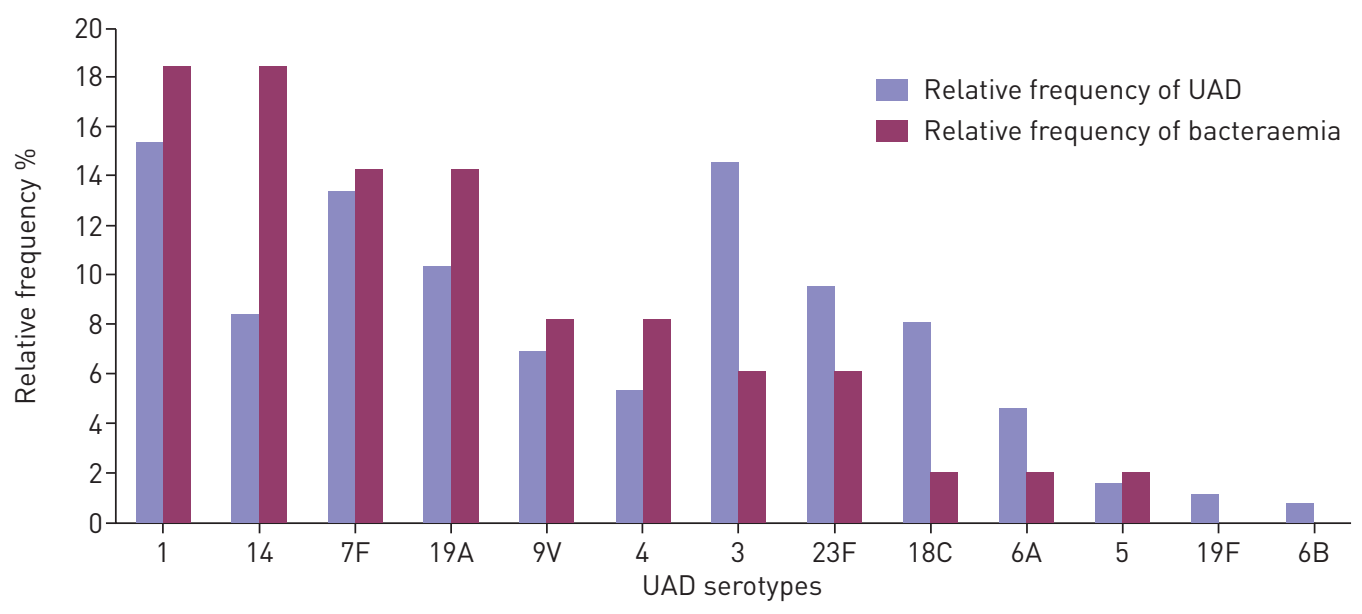

FIGURE 2 Relative serotype distribution based on urinary antigen detection (UAD) data $(n=261$, including multiple serotypes) and on bacteraemia data $(n=49)$. For absolute numbers of UAD data, see table 3 .

UAD test and one episode by three serotypes. Two of these patients also had concomitant bacteraemia and, in both cases, serotype of bacteraemia isolates matched with one of the serotypes detected by the UAD assay.

\section{Discussion}

Addition of a novel serotype-specific UAD test to commonly used standard diagnostic methods, such as microbiological cultures and ICA of urine for pneumococcal antigens, increased the diagnostic yield for S. pneumoniae CAP by $39 \%$. The sensitivity of the UAD test was $98 \%$ for pneumococcal CAP episodes caused by one of the serotypes included in the UAD test, as compared with $69 \%$ for ICA. Specificity for detecting 13 serotype-specific pneumococcal antigens in the urine of adult patients with CAP was $100 \%$, and similar to the ICA test. Moreover, $4 \%$ of pneumococcal CAP episodes diagnosed by the UAD test resulted from co-infection with multiple serotypes. Although not commercially available, this approach could enhance our diagnostic yield for pneumococcal infections and could provide more detailed information on the epidemiology of infection-associated pneumococcal serotypes.

Detection of pneumococcal antigen in urine was first described in 1917 [18], and became widely applied as of 1999 with the commercial availability of the ICA BinaxNOW (Alere International). Serotype-specific antigen detection in urine has been attempted before, with excellent specificity (98-99\%), but disappointing sensitivity (55-84\%), when compared with S. pneumoniae isolates detected in sterile sites [19-22]. The sensitivity of the ICA test in the present study was $63 \%$.

\section{TABLE 3 Serotype distribution based on urinary antigen detection results}

$\begin{array}{cc}\text { Frequencies } & \text { Frequencies } \\ \text { lonly single serotypes) } & \text { (including multiple serotypes) }\end{array}$

\begin{tabular}{lcc}
\hline $\mathbf{1}$ & 38 & 40 \\
$\mathbf{3}$ & 35 & 38 \\
$\mathbf{7 F}$ & 32 & 35 \\
$\mathbf{1 9 A}$ & 24 & 27 \\
$\mathbf{1 4}$ & 22 & 22 \\
$\mathbf{2 3 F}$ & 21 & 25 \\
$\mathbf{1 8 C}$ & 18 & 21 \\
$\mathbf{9 V}$ & 16 & 18 \\
$\mathbf{4}$ & 14 & 14 \\
$\mathbf{6 A}$ & 10 & 12 \\
$\mathbf{5}$ & 3 & 4 \\
$\mathbf{1 9 F}$ & 3 & 3 \\
$\mathbf{6 B}$ & 2 & 2 \\
Total & 238 & 261 \\
\hline
\end{tabular}

Data are presented as $\mathrm{n}$. 
With the addition of the UAD test to the conventional diagnostic methods, the proportion of CAP episodes caused by S. pneumoniae increased from $23.3 \%$ to $32.6 \%$. However, the UAD test detects only 13 serotypes and there were 71 pneumococcal CAP episodes diagnosed through a positive ICA result only. Extrapolation of the observed $63 \%$ sensitivity of the ICA test for bacteraemic CAP would imply that there were 113 instead of the observed 71 additional episodes of pneumococcal CAP caused by serotypes not covered by the UAD test. That would imply that the total proportion of CAP episodes caused by pneumococci would be $36.4 \%$ (399 out of 1095). The UAD test as used in this study "serotyped" three times more episodes of pneumococcal CAP (249 compared with 77 for isolates from sterile sites and sputum) and, therefore, enhances our capacity to investigate the serotype-epidemiology of S. pneumoniae. Moreover, if the turnaround time of this test could be reduced to that of a real-time test, demonstrating $S$. pneumoniae as a cause of CAP could assist in rapid de-escalation of unnecessarily broad empirical antibiotic treatment for CAP. As many serotypes will not be detected, a negative test result will not be useful for clinical decision-making.

Of the pneumococcal CAP cases detected by the UAD test, $4.4 \%$ appeared to be caused by multiple serotypes. Although the presence of carriage with multiple serotypes has been described [23-25], infections caused by multiple serotypes have been reported only sporadically [20, 21, 26, 27]. Recently, five out of 366 patients with pneumococcal CAP had pneumonia caused by two serotypes based on a multiplex immunoassay for 14 serotypes in urine samples [28]. Thus, apart from higher sensitivity, the UAD test also improves our capacity to detect co-infections with multiple serotypes.

Our study has some limitations. As in all clinical studies investigating the diagnosis of noninvasive S. pneumoniae infections, we suffered from the absence of a true "gold standard". This results in low numbers of "true positives" ( $n=49)$ and "true negatives" $(n=76)$. However, at this point, there is no other reference standard to better test accuracy of serotype specific diagnostic tools. Secondly, pneumococcal antigens can persist in urine samples after infection for periods as long as from 7 days [19,29] to 3 months [30]. Although we did not have detailed information about disease history in the weeks before the clinical episode of CAP, we could determine that only eight $(0.7 \%)$ patients had been hospitalised in the weeks before the CAP episode. Moreover, persistent antigen positivity is more likely when concentrated urine is used $[11,30]$. In our study, only unconcentrated urine was used, reducing the possibility of false positive tests. Therefore, we consider it unlikely that false-positive test results due to previous pneumococcal infections influenced our findings. Finally, the absence of a urine sample in 101 patients could have created a selection bias. Indeed, these 101 patients were more frequently categorised as "severe CAP" (PSI class V) $(p=0.012$; data not shown $)$ and previous studies have suggested that the ICA is more sensitive in severely ill patients [21,31], although this finding was not confirmed by others [10,32]. If severity of CAP is associated with a higher likelihood of antigen detection in urine, the diagnostic yield of the UAD test might actually be higher than reported here.

\section{Acknowledgements}

The authors would like to acknowledge the substantial contribution to this study made by Marcel Peeters (St Elisabeth Hospital, Tilburg, the Netherlands), who sadly passed away in June 2011. Furthermore, they would like to thank all CAP diagnostic investigators for their time and effort.

\section{References}

1 Woodhead MA, Macfarlane JT, McCracken JS, et al. Prospective study of the aetiology and outcome of pneumonia in the community. Lancet 1987; 1: 671-674.

2 Jokinen $\mathrm{C}$, Heiskanen L, Juvonen $\mathrm{H}$, et al. Microbial etiology of community-acquired pneumonia in the adult population of 4 municipalities in eastern Finland. Clin Infect Dis 2001; 32: 1141-1154.

3 Hausdorff WP, Bryant J, Paradiso PR, et al. Which pneumococcal serogroups cause the most invasive disease: implications for conjugate vaccine formulation and use, part I. Clin Infect Dis 2000; 30: 100-121.

4 Harboe ZB, Thomsen RW, Riis A, et al. Pneumococcal serotypes and mortality following invasive pneumococcal disease: a population-based cohort study. PLoS Med 2009; 6: e1000081.

5 Campbell SG, Marrie TJ, Anstey R, et al. The contribution of blood cultures to the clinical management of adult patients admitted to the hospital with community-acquired pneumonia: a prospective observational study. Chest 2003; 123: 1142-1150.

6 van de Garde EM, Endeman H, van Hemert RN, et al. Prior outpatient antibiotic use as predictor for microbial aetiology of community-acquired pneumonia: hospital-based study. Eur J Clin Pharmacol 2008; 64: 405-410.

7 Metersky ML, Ma A, Bratzler DW, et al. Predicting bacteremia in patients with community-acquired pneumonia. Am J Respir Crit Care Med 2004; 169: 342-347.

8 Rosón B, Carratalà J, Verdaguer R, et al. Prospective study of the usefulness of sputum Gram stain in the initial approach to community-acquired pneumonia requiring hospitalization. Clin Infect Dis 2000; 31: 869-874.

9 Ewig S, Schlochtermeier M, Goke N, et al. Applying sputum as a diagnostic tool in pneumonia: limited yield, minimal impact on treatment decisions. Chest 2002; 121: 1486-1492.

10 Gutiérrez F, Masiá M, Rodríguez JC, et al. Evaluation of the immunochromatographic Binax NOW assay for detection of Streptococcus pneumoniae urinary antigen in a prospective study of community-acquired pneumonia in Spain. Clin Infect Dis 2003; 36: 286-292. 
Marcos MA, Jiménez de Anta MT, de la Bellacasa JP, et al. Rapid urinary antigen test for diagnosis of pneumococcal community-acquired pneumonia in adults. Eur Respir J 2003; 21: 209-214.

12 Domínguez J, Galí N, Blanco S, et al. Detection of Streptococcus pneumoniae antigen by a rapid immunochromatographic assay in urine samples. Chest 2001; 119: 243-249.

13 Hak E, Grobbee DE, Sanders EA, et al. Rationale and design of CAPITA: a RCT of 13-valent conjugated pneumococcal vaccine efficacy among older adults. Neth J Med 2008; 66: 378-383.

14 Pride MW, Huijts SM, Wu K, et al. Validation of an immunodiagnostic assay for detection of 13 Streptococcus pneumoniae serotype-specific polysaccharides in human urine. Clin Vaccine Immunol 2012; 19: 1131-1141.

15 Austrian R. The quellung reaction, a neglected microbiologic technique. Mt Sinai J Med 1976; 43: 699-709.

16 Lund E, Henrichsen J. Laboratory diagnosis, serology and epidemiology of Streptococcus pneumoniae. Methods Microbiol 1978; 12: 241-262.

17 Fine MJ, Auble TE, Yealy DM, et al. A prediction rule to identify low-risk patients with community-acquired pneumonia. N Engl J Med 1997; 336: 243-250.

18 Dochez AR, Avery OT. The elaboration of specific soluble substance by pneumococcus during growth. $J$ Exp Med 1917; 26: 477-493.

19 Leeming JP, Cartwright K, Morris R, et al. Diagnosis of invasive pneumococcal infection by serotype-specific urinary antigen detection. J Clin Microbiol 2005; 43: 4972-4976.

20 Scott JA, Hannington A, Marsh K, et al. Diagnosis of pneumococcal pneumonia in epidemiological studies: evaluation in Kenyan adults of a serotype-specific urine latex agglutination assay. Clin Infect Dis 1999; 28: 764-769.

21 Stralin K, Kaltoft MS, Konradsen HB, et al. Comparison of two urinary antigen tests for establishment of pneumococcal etiology of adult community-acquired pneumonia. J Clin Microbiol 2004; 42: 3620-3625.

22 Sheppard CL, Harrison TG, Smith MD, et al. Development of a sensitive, multiplexed immunoassay using xMAP beads for detection of serotype-specific Streptococcus pneumoniae antigen in urine samples. J Med Microbiol 2011; 60: 49-55.

23 da Gloria Carvalho M, Pimenta FC, Jackson D, et al. Revisiting pneumococcal carriage by use of broth enrichment and PCR techniques for enhanced detection of carriage and serotypes. J Clin Microbiol 2010; 48: 1611-1618.

24 Huebner RE, Dagan R, Porath N, et al. Lack of utility of serotyping multiple colonies for detection of simultaneous nasopharyngeal carriage of different pneumococcal serotypes. Pediatr Infect Dis J 2000; 19: 1017-1020.

25 Kaltoft MS, Skov Sørensen UB, Slotved HC, et al. An easy method for detection of nasopharyngeal carriage of multiple Streptococcus pneumoniae serotypes. J Microbiol Methods 2008; 75: 540-544.

26 Brandileone MCDC, Vieira VSD, Casagrande ST, et al. Prevalence of serotypes and antimicrobial resistance of Streptococcus pneumoniae strains isolated from Brazilian children with invasive infections. Pneumonococcal Study Group in Brazil for the SIREVA Project. Regional System for Vaccines in Latin America. Microb Drug Resist 1997; 3: $141-146$.

27 Cockrell SL, Ruegsegger JM. Multiple pneumococcus infections in pneumonia. J Infect Dis 1941; 68: 33-36.

28 Bewick T, Sheppard C, Greenwood S, et al. Serotype prevalence in adults hospitalised with pneumococcal noninvasive community-acquired pneumonia. Thorax 2012; 67: 540-545.

29 Smith MD, Derrington P, Evans R, et al. Rapid diagnosis of bacteremic pneumococcal infections in adults by using the Binax NOW Streptococcus pneumoniae urinary antigen test: a prospective, controlled clinical evaluation. $J$ Clin Microbiol 2003; 41: 2810-2813.

30 Andreo F, Prat C, Ruiz-Manzano J, et al. Persistence of Streptococcus pneumoniae urinary antigen excretion after pneumococcal pneumonia. Eur J Clin Microbiol Infect Dis 2009; 28: 197-201.

31 Hohenthal U, Vainionpää R, Meurman O, et al. Aetiological diagnosis of community acquired pneumonia: utility of rapid microbiological methods with respect to disease severity. Scand J Infect Dis 2008; 40: 131-138.

32 van der Eerden MM, Vlaspolder F, de Graaff CS, et al. Value of intensive diagnostic microbiological investigation in low- and high-risk patients with community-acquired pneumonia. Eur J Clin Microbiol Infect Dis 2005; 24: 241-249. 